\title{
OSTEOCHONDRITIS DISSECANS OF THE KNEE IN CHILDREN AND ADOLESCENTS: OUR EXPERIENCE WITH TRANSCHONDRAL DRILLING
}

\author{
Haroun Hassan Shaikh, Jan Vícha, Tomáš Proček, Jaroslav Pavlata, Tomáš Kučera
}

\begin{abstract}
Charles University in Prague, Faculty of Medicine and University Hospital in Hradec Králové, Czech Republic: Department
\end{abstract} of Orthopedic Surgery

Summary: Osteochondritis dissecans(OCD) of the knee is identified with increasing frequency in the adolescent patient. Left untreated, OCD can cause significant impairment and restriction in physical activity and development of osteoarthritis at an early age. The diagnosis of lesions of OCD can be confirmed on plain radiographs. MRI has emerged as the gold standard to evaluate the stability of the lesion and the integrity of the overlying articular cartilage. Treatment of OCD lesions depend on the stability of the lesion. Stable lesions can be treated conservatively by physical activity modification and immobilization. Unstable lesions and stable lesions not responding to conservative measures should be treated surgically. Surgical options range from arthroscopic drilling, either transarticular or extra-articular drilling for stable lesions or salvage procedures such as autologous chondrocyte transplantation (ACT), mosaicplasty to restore joint and cartilage congruency.

Keywords: Osteochondritis dissecans; Juvenile OCD; Transarticular drilling; Knee joint

\section{Introduction}

Osteochondritis dissecans (OCD) is a condition that affects the subchondral bone and the articular cartilage and can lead to separation of articular fragment from the underlying bone. The Research for Osteochondritis dissecans of the knee (ROCK) recently defined OCD as a focal idiopathic alteration of subchondral bone with risk for instability and disruption of adjacent articular cartilage that may result in premature osteoarthritis (12). The term osteochondritis dissecans was first coined by Konig in 1888 , describing it as an inflammation of the bone-cartilage interface (16). Though it remains a well known condition, the cause is still unknown but trauma, ischaemia, defects in ossification and genetic causes have all been suggested (14). Classification of OCD can be based on age, location, radiographic and MRI findings, and intraoperative appearance. OCD is classified as a juvenile or adult form based on the skeletal maturity of the patiens (5). Juvenile OCD occurs in children and adolescents with open growth plates. Juvenile OCD has a much better prognosis than does adult OCD, with higher rates of spontaneous healing with nonoperative treatment (3). Although the majority of OCD lesions occur on the medial femoral condyle, they can occur anywhere.

Subchondral drilling for creation of revascularization channels is the primary surgical option used for treating juvenile OCD lesions. Standard drilling techniques either involve antegrade (transarticular) penetration of the intact articular cartilage or extra-articular (retrograde) approaches preserving the cartilage surface. The aim of this study is to review a total of 17 cases of juvenile OCD of the femoral condyle of the knee treated by transarticular drilling technique.

\section{Materials and methods}

The retrospective study included all patients with juvenile OCD operated between 2007 and 2014 in the orthopaedic department of our university hospital.

The records of all patients, between the age group of 5 to 17 , diagnosed and surgically treated for juvenile OCD between 2007 and 2014 were reviewed. The following data were collected for each patient: demographics( age at diagnosis, gender), time between the conformation of diagnosis and operation, clinical finding, intensity of pain at admission graded from 0-10 according to the visual analog scale (VAS), plain radiograph films (antero-poterior and lateral views). All but two patients demonstrated radiographic evidence of juvenile OCD on plain radiographs, an MRI was carried out in the patients in whom radiographically the lesion was in early stages. There were a total of 8 boys and 9 girls with a mean age of 12.3 years. They were followed up for a mean period of 2.2 years. The average age at the time of surgery was 13.2 years. The mean time interval between the first clinical examination to the time of operation was 4.6 months and the mean duration of symptoms was 4.8 months. The affected knee, femoral condyles, preoperative Lysholm score, radiograph findings, arthroscopic 


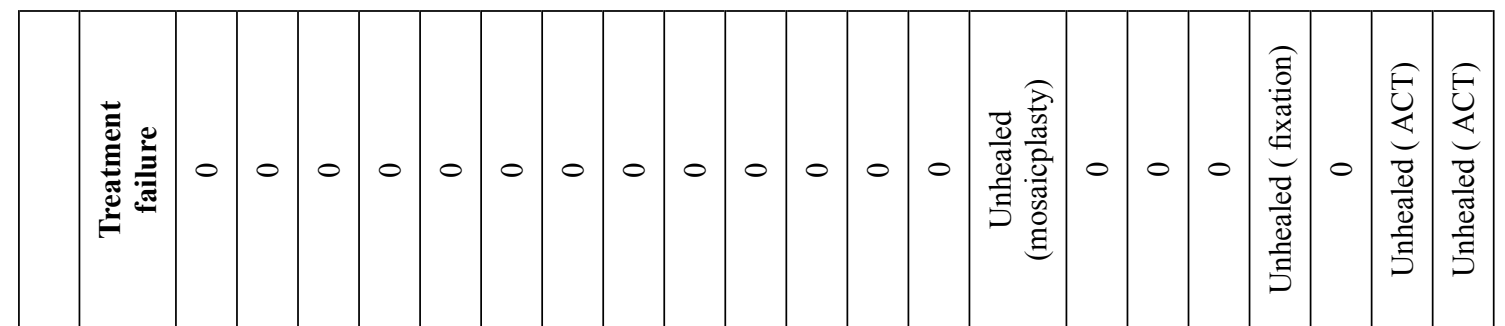

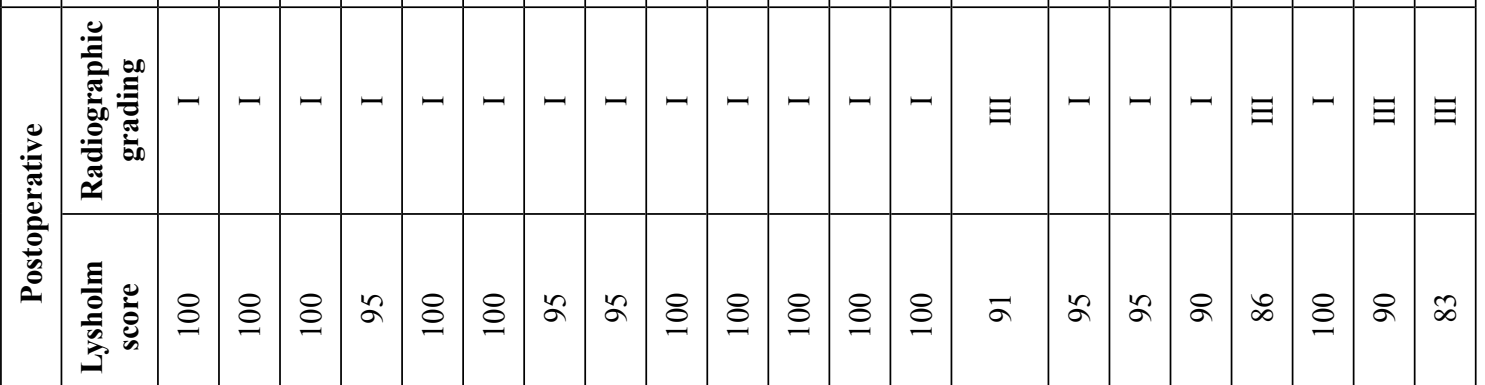

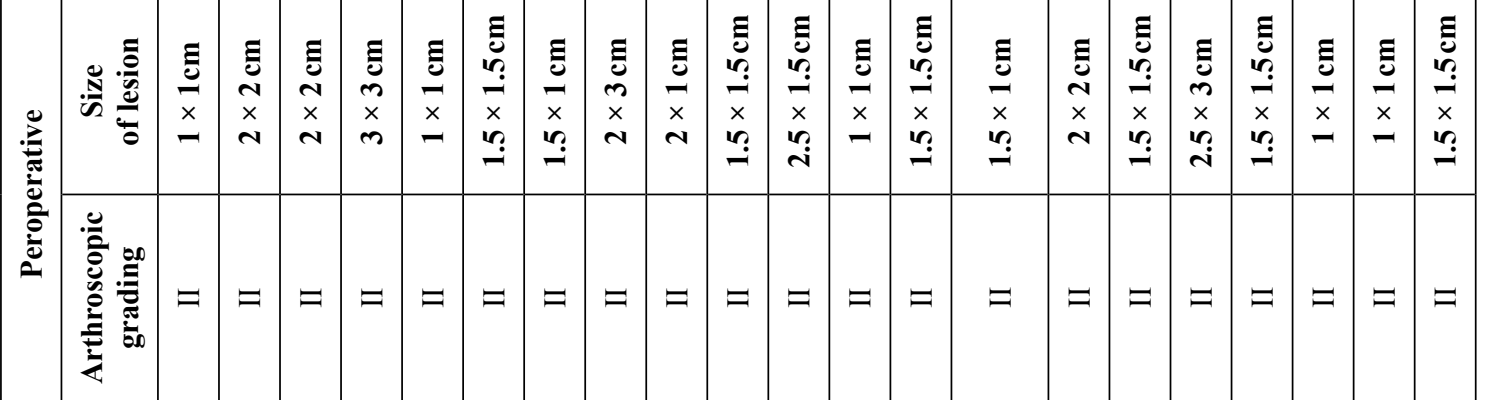

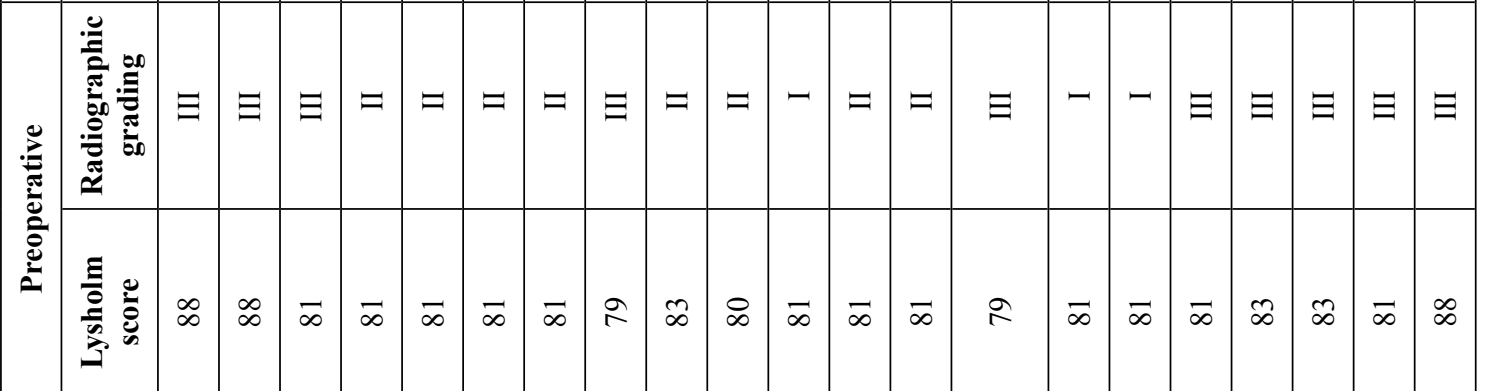

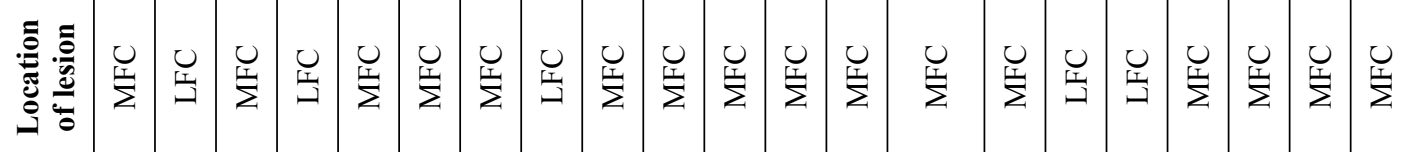

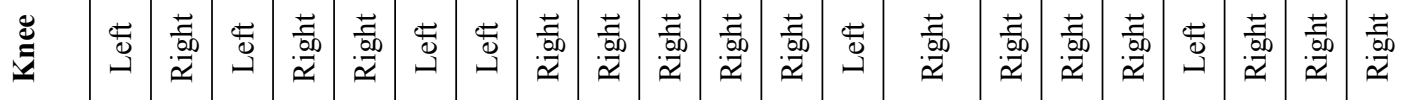

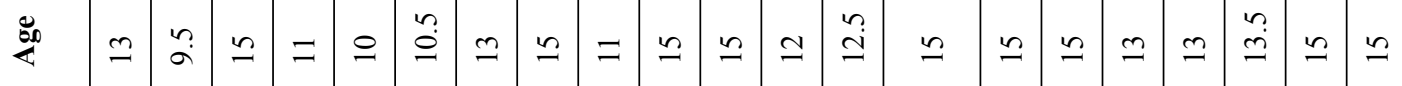

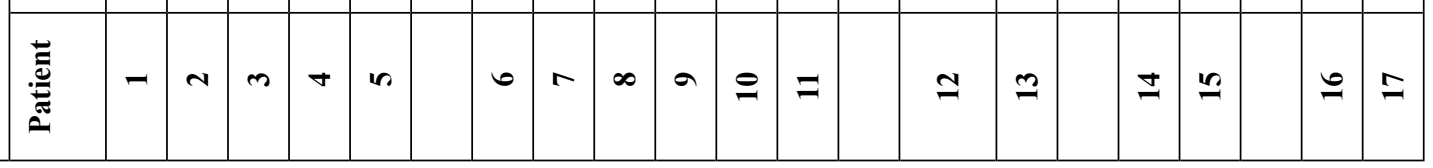

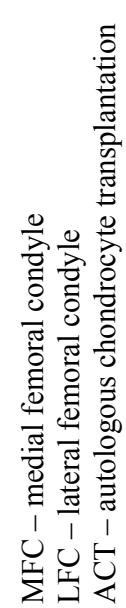


Tab. 2: Grading of osteochondral lesions arthroscopically and radiographically.

\begin{tabular}{|c|l|l|}
\hline \multicolumn{2}{|c|}{ Staging system for characterizing osteochondral lesions } \\
\hline Grade & Arthroscopic (Guhl) & Radiographs \\
\hline I & $\begin{array}{l}\text { Irregularity and softening of articular Cartilage.No } \\
\text { definable fragment }\end{array}$ & No findings \\
\hline II & $\begin{array}{l}\text { Articular cartilage breached, definable fragment, not } \\
\text { displaceable }\end{array}$ & Subchondral radiolucency \\
\hline III & $\begin{array}{l}\text { Articular cartilage breached, definable fragment, } \\
\text { Displaceable, but attached by some overlying articular } \\
\text { cartilage }\end{array}$ & $\begin{array}{l}\text { Well circumscribed area of subchondral bone separated by } \\
\text { a sclerotic radiolucent outline of fragment }\end{array}$ \\
\hline IV & Loose body & Loose body \\
\hline
\end{tabular}

findings and the postoperative Lysholm score and radiograph findings are detailed in Table 1.

On presentation a detailed physical examination was carried out which included range of movement of the knee joint, effusion of knee joint, quadriceps atrophy, presence of knee locking. The radiograph findings were graded from I to IV) (Table 2) (7).

All patients were advised non weight bearing of the affected side on crutches till the period of arthroscopy.

Arthroscopy had been taken under general or spinal anesthesia. No preventive antibiotics were administered during the operative procedure. Patients were operated with the use of a thigh tourniquet. Standard medial and lateral parapatellar portals were used. The articular cartilage was carefully visualized and probed to determine the extent of loosening at the site of lesion. The OCD lesion was then graded according to the classification by Guhl (9) (Table 2). All lesions were treated with transarticular drilling. For lesions of the lateral aspect of the medial femoral condyle, the medial portal is used as the viewing port and the lateral portal is used as the working portal and vice versa in lesions of the lateral femoral condyle. All lesions are drilled into the adjacent subchondral bone to a depth of $2 \mathrm{~mm}$ with a $1.2 \mathrm{~mm}$ smooth Kirschner wire to encourage bleeding at the base of the lesion and healing. The number of holes drilled depended on the size of the lesion( on an average between three and six). The number of holes drilled depended on the peroperative findings. What was more important, was that the holes were drilled 3 to $4 \mathrm{~mm}$ apart, while taking care not to damage the subchondral plate between the holes.

Postoperatively all patients were advised non weight bearing for a period of 6 weeks but immediate range of movement is allowed. Weight bearing was then gradually advanced but activities were still limited for a period of 3 months postoperatively. Sport activites were permitted after a period of 6 months.

\section{Postoperative Evaluation}

Postoperative evaluation included clinical and radiographic examination at follow-up visits to monitor healing. Radiographs included AP, lateral views. In one case an MRI and in another case a CT was carried out to determine extent of healing. Healing on radiographs was defined as disappearance of the radiolucent zone or union of the displaced fragment. We used the Lysholm score to assess improvement in knee function. In addition, clinical improvement in symptoms was measured using VAS.

\section{Results}

Out of the 17 patients, 7 players were involved in active sporting activities like football or judo, interestingly 3 patients with lateral femoral condyle lesions had previously undergone arthroscopy of the knee for modellation of lateral discoid meniscus, 1 patient had habitual subluxation of patella, and the remaining were not involved in any sporting activities. The most common complaint was poorely localised knee pain, no patient presented with knee effusion, quadriceps atrophy.

OCD was diagnosed in 19 of the 21 knees from plain $\mathrm{X}$-ray films alone, while in the remaining two knees an MRI was carried out in the patients in whom radiographically the lesion was in early stages. We classified the lesions radiographically as well as arthroscopically accorging to Guhl (9) (Table 2). Interestingly, one patient had OCD of both the femoral condyles of the right knee.

Postoperatively, there was an overall improvement in Lysholm knee score from a mean preoperative score of 82.2 to a mean postoperative score of 96 . Radiographically, 17 of grade III or grade II lesions improved to grade I lesion, while the remaining four grade III lesions remained stationary and had to undergo further procedures. In four patients despite showing no radiographic signs of improvement, there was an improvement in VAS and Lysholm scores.

The visual analog score was used to measure the subjective improvement in symptoms. There was a significant decrease in VAS pre and postoperatively from 5.45 to 1.55 . No post-operative infection or thromboflebitis occured. The only complication that was encountered were unhealed lesions following transarticular drilling. In our study one patient had to undergo mosaicplasty, 2 patients had to ACT, while one patient had to undergo fixation of the fragment with the help of Herbert screws for no radiographic signs of 


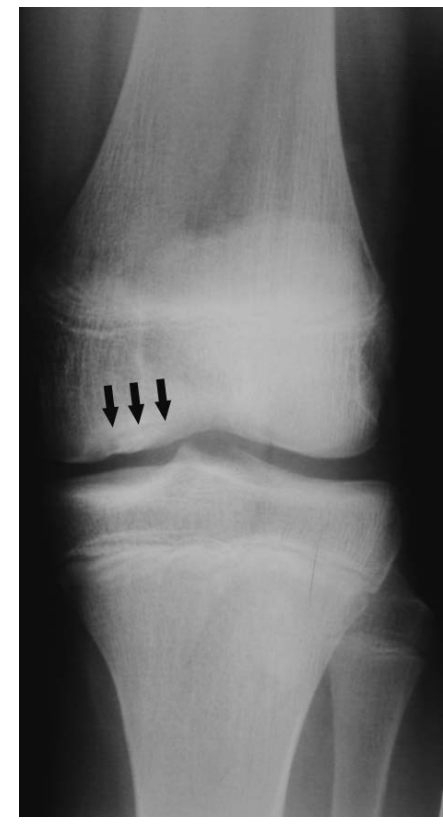

Fig. 1:

Pre-operative AP view of the knee showing an $\mathrm{OCD}$ lesion (black arrows).

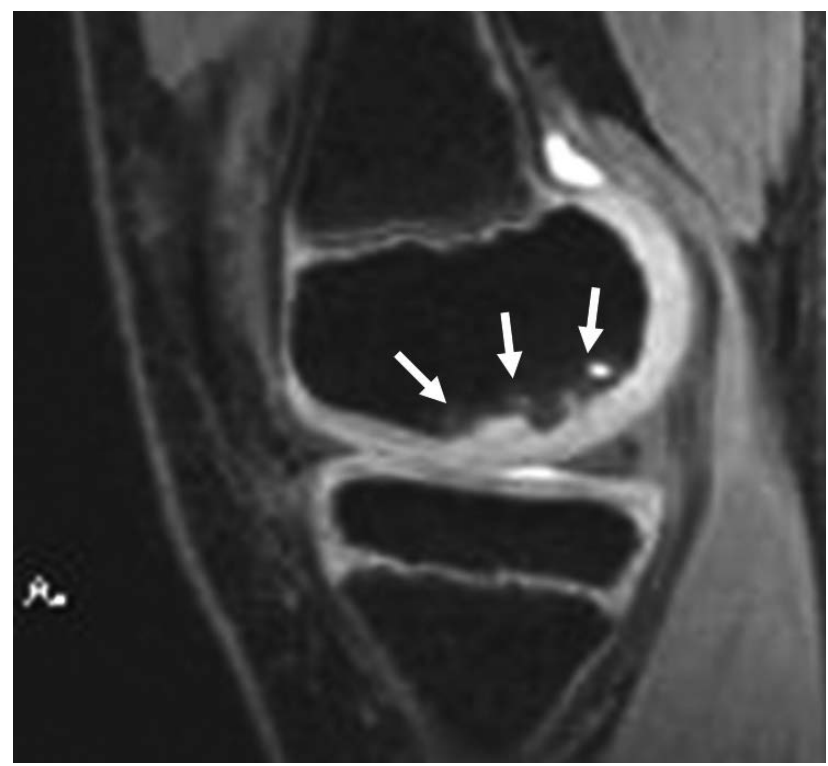

Fig. 3: MRI - sagittal section showing OCD lesion (white arrows).

healing. All these patients after the above mentioned operations had satisfactory results.

\section{Discussion}

OCD is a localized condition of subchondral bone and overlying cartilage most commonly occuring in the knee. Possible causative factors of OCD include repetitive microtrauma, ischemia, genetic and endocrine factors and anomalies of ossification (16). A multicenter study carried out by the European Pediatric Orthopaedic Society showed

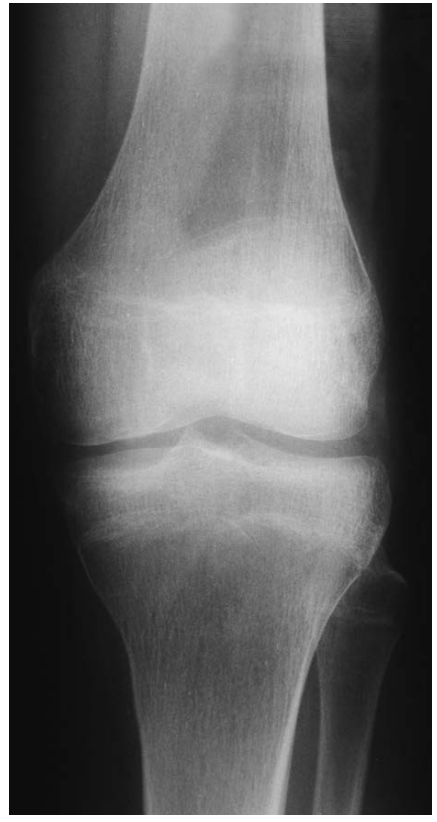

Fig. 2:

Post-operative AP view of the knee 5 months after transarticular drilling demonstrating completely healed OCD lesion.

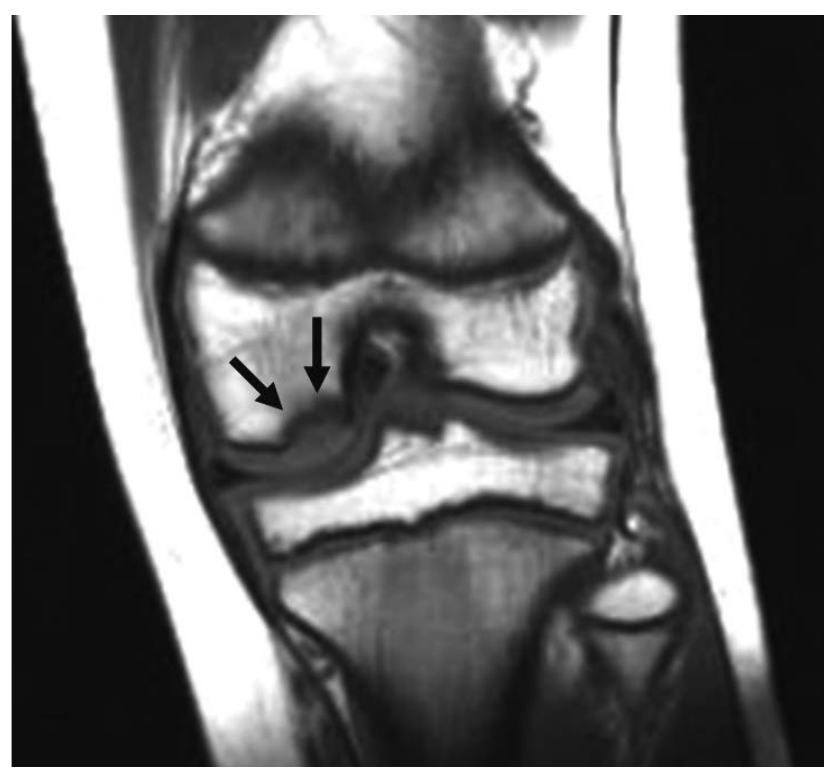

Fig. 4: MRI - frontal section showing OCD lesion (black arrows).

that nearly $55 \%$ of the patients with OCD were either regularly active in sports or performed strenous athletic activity (11). OCD most commonly affects the medial femoral condyle, rarely the lateral femoral condyle is affected. Interestingly, in our study one patient had OCD lesion of both the femoral condyles. Hanna et al. (10) also reported two cases of bicondylar OCD of the knee. Prior to their study no case of bicondylar OCD of the knee had been reported. Furthermore, the presence of discoid meniscus has also been reported to play a role in the development of OCD. Discoid menisci are abnormal in macroscopic and microscopic struc- 
ture. Their ability to provide normal articular surface and subchondral region load sharing and stress shielding has not been documented. Alterations of joint mechanics produced by a tear of the discoid meniscus with fragment instability and compromised joint function may produce increases in peak loading forces such as those documented following compromise of menisci of normal morphology. This modification may allow repetitive micro-trauma to summate in forces that produce a subchondral lesion.

Treatment of OCD depends on age of presentation, fragment size, fragment location and fragment stability. Stable lesions in skeletally immature patients are more amenable to conservative management (19). The disruption of subchondral blood supply is an important factor in the development of OCD (20). Fragment healing is enhanced by creating vascular channels to the devitalized region, also known as drilling. Today arthroscopic or open drilling, either alone or with various fixation methods, remains one of the most common operative interventions. Open drilling and reduction was first proposed by Smillie in 1957 (18), since then many authors have suggested drilling alone or with various fixation methods and it still remains the most common operative procedure for OCD lesions. Multiple authors have reported high rates of healing with arthroscopic drilling. Kocher et al. (13) reported $100 \%$ radiographic healing in 30 skeletally immature knees treated with transarticular arthroscopic drilling after an average of 4.4 months, and Aglietti et al. (1) and Bradley and Dandy (4) reported $95 \%$ and $82 \%$ radiographic healing, respectively.

An alternative method is retrograde drilling, which does not violate the intact articular cartilage. However, retrograde drilling is a technically demanding operation that requires fluoroscopy (2).

Irrespective of the drilling technique used, drilling still remains an effective treatment option for stable OCD lesions, especially in patients with open physes who tend to have greater healing potential.

A variety of treatments have evolved over the past few years aimed at addressing irreparable chondral defects and OCD lesions. These include microfracture, autologous chondrocyte transplantation, mosaicplasty. These techniques are reserved for unsalvageble lesions such as those with extensive comminution, fragmentation or with chronic complete detachent of the chondral lesion. Newer surgical treatments, including single-stage cell-based procedures, use mesenchymal stem cells and matrix augmentation (8).
The treatment of osteochondral lesions in the knee is dictated by the stability of the osteochondral fragment and thus the overlying cartilage. Though standard anteroposterior and lateral radiographs of the knee permit localalization of the lesion, they however do not permit visualization of the articular cartilage and do not indicate whether there is fibrous attachment of the osteochondral fragment to the underlying bone. Furthermore, most the the times it is difficult to distinguish between grade II and grade III lesions on plain radiograph films.

Recent studies have advocated the use of MRI as an accurate method of preoperative staging for osteochondral lesions. A classification system has been devised by Dipaola et al. (6) to stage OCD on MRI (Table 3).

The most useful diagnostic feature of MRI is the ability to distinguish between Stage II and Stage III lesion. In Stage II lesion with stable fibrous attachment, a heterogenous area of mixed higher and lower signal intensity is seen behind the lesion. In contrast, Stage III lesions will show a thin, homogenus intermediate signal behind the lesion, indicating synovial fluid between the fragment and underlying subchondral bone. The ability to distinguish between Stage II and Stage III lesions preoperatively is useful in planning surgery. Stage II lesions are stable and have mostly intact articular cartilage, which can be treated by drilling for revascularisation of the fragment. Stage III lesions are unstable and require fixation in addition to drilling to maximize chances of healing.

A recent study by O'Connor et al. (15) has further improved the accuracy of MRI staging the OCD lesion from $45 \%$ to $85 \%$ by interpretating the high signal $\mathrm{T} 2$ line as a predictor of instability only when it was accompanied by a breach in the cartilage on the T1-weighted image. According to this study the appearance of a high signal line on T2-weighted image does not mean a poor prognosis for the lesion. This high signal line may represent vascular granulation tissue and hence a healing response. Only if this high signal line is associated with a break in the articular cartilage, seen on T1-weighted image, it can be said to represent synovial fluid. If there is no such breach the lesion can be treated conservatively since healing may occur without surgery.

Currently, MRI is the preferred imaging modality for both diagnosis and the assessment of healing potential of OCD of the knee due to its ability to provide excellent anatomical detail of both the bone and soft tissue structures with the absence of harmful ionizing radiation (17).

Tab. 3: Classification of OCD lesions on MRI.

\begin{tabular}{|l|l|}
\hline \multicolumn{2}{|l|}{ Staging system for characterizing osteochondral lesions } \\
\hline Stage I & Thickening of articular cartilage and low signal changes \\
\hline Stage II & Articular cartilage breached, low signal rim behind fragment indicating fibrous attachment \\
\hline Stage III & $\begin{array}{l}\text { Articular cartilage breached, high signal changes behind fragment indicating synovial fluid between fragment and } \\
\text { underlying subchondral bone }\end{array}$ \\
\hline Stage IV & Loose body \\
\hline
\end{tabular}




\section{Conclusion}

In conclusion OCD of the knee should be considered to a differential diagnosis among children with acute or subactue knee pain. The history, physical examination, imaging examinations (especially MRI) and arthroscopy are important for the early diagnosis and conservative or surgical management of the process aimed at preserving the cartilage and joint congruence. Untreated or inadequately treated lesions may progress from stable, intact lesions to unstable lesions with loose body formation, chondral tears, and full thickness defects with underlying loose fibrous tissue. Allowing these patients to continue their acitivities unchanged despite intermittent pain, mechanical symptoms and radiographic changes is to allow patient to potentially progress to significant early gonarthrosis. Reestablishment of the joint surface, improvement of the fragment's blood supply, rigid fixation, and early motion are the primary goals for osteochondral fragment preservation. When the fragement is not suitable for preservation, careful consideration of defect location and the patient's clinical presentation will determine when cartilage restoration procedures should be performed. Successful restorative options should relieve pain, restore function and prevent the development of secondary gonarthrosis.

For the future, based on the retrospective study of our pateints with JOCD lesions we have come to two main concluions. Firstly, every patient diagnosed with OCD must undergo MRI, to enable proper preoperative staging of OCD lesions and then depending on the grade of lesion, plan proper treatment. Secondly, all grade I or grade II lesions, can be treated conservatively for a minimum period of six months after which a control MRI should be cariried out. In our study we were not able to differentiate between grade II and grade III lesions on X-rays which lead to unfavourable results in two cases, we therefore recommend preoperative MRI of every OCD lesion for proper grading of the lesion and subsequent planning of treatment. If there are no signs of healing or if the lesion is unstable, then the lesion is ought to be treated surgically, either just drilling or fixation in addition to drilling to maximize the chances of healing.

\section{Acknowledgements}

This work was supported by the programme PRVOUK P37/04 and by MHCZ-DRO (UHHK, 00179906).

\section{References}

1 Agletti P, Buzzi R, Bassi PB, et al. Arthroscopic drilling in juvenile osteochondritis of the medial femoral condyle. Arthroscopy 1994; 10: 286-291.

2. Boughanem J, Riaz R, Patel RM, Sarwark JF. Functional and radiographic outcomes of juvenile osteochondritis dissecans of the knee treated with extra-articular retrograde drilling Am J Sports Med 2011; 39(10): 2212-7.

3. Bradley J, Dandy DJ. Osteochondritis dissecans and other lesions of the femoral condyles. J Bone Joint Surgery Br 1989; 71(3): 518-522.

4. Bradley J, Dandy DJ. Results of drilling osteochondritis dissecans before skeletal maturity. J Bone Joint Surgery Br 1989; 71: 642-644.

5. Cahill BR. Osteochondritis dissecans of the knee: treatment of juvenile and adult forms. J Am Acad Orthop Surg 1995; 3(4): 237-247.

6. Dipaola J, Nelson DN, Colville MR. Charecterising osteochonral lesions by magnetic resonance imaging. Arthroscopy 1991; 7: 101-104.

7. Book: Dungl P et al. (2005) Ortopedie. Prague, Grada publishing.

8. Erickson BJ, Chambers PN, Yanke AB, et al. Surgical management of osteochondritis dissecans of the knee. Curr Rev Musculoskelet Med 2013; 6: 102-114.

9. Guhl JF. Arthroscopic treatment of osteochondritis dissecans. Clin Orthop 1982; 167: 65-74.

10. Hanna SA, Aston WJS, Gikas PD, Briggs TWR. Bicondylar osteochondritis dissecans in the knee. J Bone Joint Surg Br 2008; 90-B: 232-235.

11. Hefti F, Beguiristain J, Krauspe R, et al. Osteochondritis dissecans, a multicenter study of the European Pediatric Orthopedic Society. J Pediatr Orthop B 1999; 8: 231-245.

12. Chambers HG, Shea KG, Anderson AF, et al. American Academy of Orthopaedic Surgeons Clinical Practise Guideline on: The diagnosis and Treatment of Osteochondritis Dissecans. J Bone Joint Surg Am 2012; 94(14): 1322-1324.

13. Kocher MS, Micheli LJ, Yaniv M, et al. Functional and radiographic outcome of juvenile osteochondritis of the knee treated with transarticular arthroscopic drilling. Am J Sports med 2001; 29(5): 562-566.

14. Kocher MS, Tucker R, Ganley TJ, Flynn JM. Management of Osteochondritis dissecans of the knee. Current concepts review. Am J Sports Med 2006; 34(8): 1181-1191.

15. O'Connor MA, Palaniappan M, Khan N, et al. Osteochondritis dissecans of the knee in children. A comparison of MRI and arthroscopic findings. J Bone Joint Surg Br 2002; 84: 258-262.

16. Scenck R, Goodnight JM. Osteochondritis dissecans. J Bone Joint Surg 1996; 78: 439-456.

17. Schultz JF, Chambers HG. Juvenile osteochondritis of the knee. Current concepts in diagnosis and management. Instr Course Lect 2013; 62: 455-467.

18. Smillie IS. Treatment of osteochondritis dissecans. J Bone Joint Surg Br 1957; 39 : 248-260.

19. Wall E, Von Stein D. Juvenile osteochondritis dissecans. Orthop Clin North Am 2003; 34: 341-353.

20. Williams JS Jr, Bush-Joseph CA, Bach Br Jr. Osteochondritis dissecans of the knee. Am J Knee Surg 1998; 11: 221-232.

Received: $16 / 04 / 2015$

Accepted in revised form: 09/09/2015

\section{Corresponding author:}

Haroun Hassan Shaikh, Department of Orthopedic Surgery, Teaching Hospital in Hradec Králové, Sokolská 581, Hradec Králové, Czech Republic; e-mail: haroun.shaikh@fnhk.cz 